\title{
Introduction of a new friction routine into the SWAN model that evaluates roughness due to bedform and sediment size changes
}

\author{
Grant Alexander Smith ${ }^{\text {a,* }}$, Alexander V. Babanin a ${ }^{\text {a Peter Riedel }}{ }^{\text {b }}$ I.R. Young a , \\ Stephen Oliver ${ }^{c}$, Graeme Hubbert ${ }^{c}$ \\ a Swinburne University of Technology, Melbourne, Victoria 3122, Australia \\ b Coastal Engineering Solutions, 600 Burwood Road, Hawthorn East, VIC, 3123, Australia \\ c Global Environmental Modelling Systems, PO Box 149, Warrandyte Victoria, 3113, Australia
}

\section{A R T I C L E I N F O}

\section{Article history:}

Received 27 April 2010

Received in revised form 17 November 2010

Accepted 23 November 2010

Available online 28 December 2010

\section{Keywords:}

Finite depth waves

Bottom friction

Roughness

Ripples

Lake George

Lakes Entrance

\begin{abstract}
A B S T R A C T
The significant loss of wave energy due to seabed interaction in finite depths is a known effect and bottom friction terms are used in the wave models to account for this dissipation. In this paper, a new bottominteraction function is tested by means of the SWAN model, based on measurements at two field sites, Lake George and Lakes Entrance, both in Australia. The function accounts for dependence of the friction on the formation process of bottom ripples and on the grain size of the sediment. The overall improvement of the model prediction both for the wave height and wave period is demonstrated.
\end{abstract}

Crown Copyright ( 12010 Published by Elsevier B.V. All rights reserved.

\section{Introduction}

Wave transformation in transitional and finite depth water (when depth, $d$, to wavelength, $\lambda$, ratio drops below $d / \lambda=1 / 2$ ) undergoes a dissipation and dispersion of energy that can be attributed to refraction, diffraction, shoaling, and seabed interaction. The significance of the dissipation due to seabed interaction in comparison to other dissipation processes alone has been outlined, for example, in Riedel et al. (2005), where neglecting this component in finite depths showed an overestimation of a swell wave height by a factor of two.

The interaction between the wave energy and the seabed results in a dissipation of energy that is due to bottom friction. Work is also done on bedforms both solid and mobile (cohesive and noncohesive sediments) such as sand ripples, on suspending and moving sediment, due to percolation (Komen et al., 1994), and due to excessive breaking caused by shoaling (e.g. Babanin et al., 2001). This paper is dedicated to assessing the friction caused by the roughness elements of the sea bed.

For a relatively flat granular seabed, the magnitude of the roughness that contributes to dissipation is determined by the grain size of the sediment, and dependence of the wave-motion friction on this grain size is one of the subjects of this paper. However, the bedform of a mobile

\footnotetext{
* Corresponding author. 4/11 Carramar Avenue, Camberwell 3124, Victoria, Australia. Tel.: + 61438028464.

E-mail address: grantale@hotmail.com (G.A. Smith).
}

seabed can be altered due to the action of the waves and currents. The experimental analysis of the flow in the boundary layer found, for example, eddies moving the sediment in an orbital trajectory that results in parallel ridges (or ripples). Their formation and size is determined by the dimension of the eddies (Melnikhova and Volkov, 2000). Such ripples are another subject of the paper.

The formulations investigated for this problem are described in Section 1.1. The results obtained from the implementation of the new friction subroutine are discussed in the Lake George case study in Section 2, and in a coastal application at Lakes Entrance in Section 3.

Laboratory studies undertaken using silt from Lake George, Australia, indicated that the presence of the sand ripples can increase the Nikuradse roughness, $k_{s}$, coefficient by 60 times (Babanin et al., 2005). The resulting increase in dissipation caused by the existence of the ripples can be attributed to form drag and to the vortices formed above the ripple troughs (e.g. Hsiao and Shemdin, 1978).

Although there is no theoretical model for the evaluation of the ripple geometry, empirical relationships have been devised by Miller and Komar (1980), Nielsen (1981), Sato and Mitani (1988). The corresponding roughness coefficient resulting from the ripple geometry has been investigated by Lettau (1969), Swart (1974), Grant and Madsen (1982), Raudkivi (1988), Nielsen (1992), Young and Gorman (1995), among others.

There is generally a poor correlation between predicted and measured seabed roughness, however the Nielsen (1992) method has been deemed to be one of the more accurate representations of 
reality. It accounts for both roughness due to grain size (in the absence of ripples) and roughness due to ripples. The ripple roughness is described by both height and wavelength, see Mirfenderesk (1999). This outcome has been confirmed within this paper as methods other than Nielsen (1992) that resulted in the overestimation of the Nikuradse roughness coefficient.

\subsection{Bed-form equations}

The most popular and commonly referenced method for taking into account the ripple geometry was developed by Nielsen (1981). It is an iterative procedure (see Fig. 2 in a later discussion) which estimates roughness coefficient $f_{w}$ from the ripple height and wavelength based on the Swart formula (Swart, 1976):

$f_{w}=\exp \left[5.213\left(\frac{k_{s}}{A_{b}}\right)^{0.194}-5.977\right]$.

Here, the characteristics of the ripples or the grain size are accounted for through Nikuradse roughness $k_{s}(\mathrm{~m})$ and the near-bottom excursion amplitude $A_{b}(\mathrm{~m})$. This empirical formula asymptotes at 0.3 , thus being the maximum value for $f_{w}$. The ratio of $k_{s} / A_{b}$ that produces the asymptotic limit is 0.63 . For an initially flat bed, $k_{s}$ can be determined by the grain size:

$k_{s}=2.5 D$

where $D$ is the sediment diameter $(\mathrm{m})$.

The near-bottom excursion amplitude is represented in the SWAN model as the following:

$A_{b}^{2}=2 \int_{0}^{2 \pi} \int_{0}^{\infty} \frac{1}{\sinh ^{2} k d} E(\sigma, \theta) \partial \sigma \partial \theta$

where $k$ is the wave number, $d$ is the water depth, $\sigma$ is the relative frequency, $\theta$ is the wave direction, $E(\sigma, \theta)=\sigma N(\sigma, \theta)$, where $N(\sigma, \theta)$ is termed the "wave action density".

Ripples are only present under certain conditions. When there is low energy at the seabed the sediment will be stationary, preventing the formation of ripples. When the wave energy is high (e.g. storm events), any ripples that are formed will be flattened. The Shields entrainment parameter is used to evaluate applicable ripple conditions:

$\theta_{s}=\frac{U_{b}^{2}}{(S-1) g D}$

where $U_{b}$ is the near bottom velocity ( $\left.\mathrm{m} / \mathrm{s}\right), S$ is the specific gravity of the sediment, and $g$ is the acceleration due to gravity. The near bottom

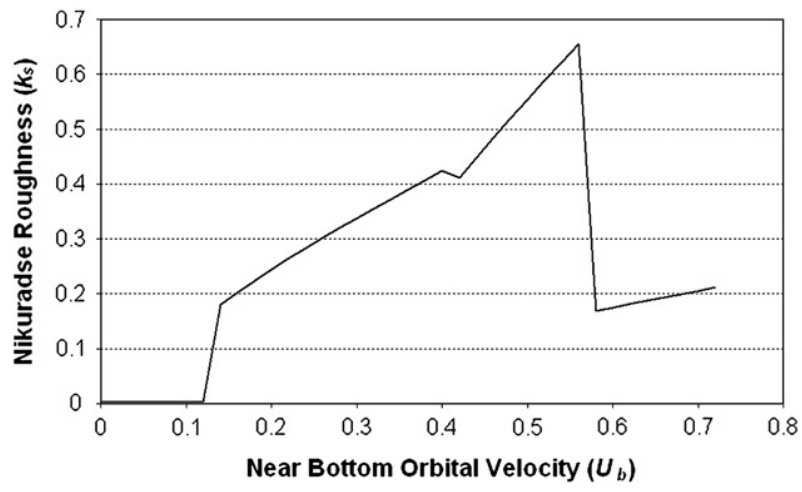

Fig. 1. Transition of the roughness coefficient as the bedform changes state (assumptions: $D=0.001, S=2.65, T_{p}=4$ ). orbital velocity is represented in the SWAN model by the following equation:

$U_{b}^{2}=\int_{0}^{2 \pi} \int_{0}^{\infty} \frac{\sigma^{2}}{g^{2} \sinh ^{2} k d} E(\sigma, \theta) \partial \sigma \partial \theta$.

Ripples are expected to generate, or evolve when $\theta_{s}$ is between 0.05 and 1. For occasions when $\theta_{s}$ is less than 0.05 , ripples will not form and therefore the Nikuradse roughness is calculated by Eq. (2). When $\theta_{s}$ is greater than $1, k_{\mathrm{s}}$ is calculated from Eq. (6):

$k_{s}=170 D \sqrt{\theta_{s}-0.05}$

Nielsen developed two sets of equations for determining ripple geometry and correspondingly roughness $k_{s}$, for both regular laboratory formed waves and irregular field waves. The mobility number is used to determine which ripple height equation to use:

$\varphi=\frac{f_{w} U_{b}}{(S-1) g D}$.

It is suggested that when the mobility number, determined from Eq. (7) is greater than 10 , then the equation for laboratory waves should be used to calculate the ripple height:

$\eta=A_{b}(0.275-0.022 \sqrt{\varphi})$.

Otherwise, the ripple height is that for irregular waves (field waves):

$\eta=A_{b} 21 \varphi^{-1.85}$

The ripple wavelength $\lambda_{r}$ is calculated from the ripple steepness, i. e. the ratio of the ripple height to the wavelength:

$\frac{\eta}{\lambda_{r}}=0.342-0.34 \theta_{s}^{0.25}$

The final step is to calculate the Nikuradse roughness for ripples which incorporates the computed ripple height and wavelength via Eq. (11). This value can then be fed back into Swart's formula (1) to obtain the friction factor, $f_{w}$.

$k_{s}=\frac{8 \eta^{2}}{\lambda_{r}}+170 D\left(\theta_{s}-0.05\right)^{0.5}$

The transition between the different bedform states can cause some discontinuities in values of the roughness coefficient in adjacent time steps in the model. In reality, the transition would be a gradual process as the bedform evolves from one state to another. Fig. 1 demonstrates the sudden roughness coefficient jumps as a function of orbital velocity. To emulate the gradual transitional process that occurs, a roughness averaging technique was employed (as discussed in Section 2.3).

The objective in this paper is to describe the implementation of an additional formulation within the SWAN model that evaluates whether ripples are expected to occur at each time step, and what the associated friction coefficient would be for the particular bedform. If there are no ripples, the formulation incorporates the friction coefficient based on the pre-defined grain size. The new algorithm will be validated by comparing model results with measurements at Lake George and Lakes Entrance.

\subsection{The SWAN model}

Modelling the bottom friction in this study will be done by means of the SWAN model. The SWAN numerical model (Simulating WAves Nearshore) is a third-generation wave model which was designed specifically for finite-depth applications (Booij et al., 1999). "The 


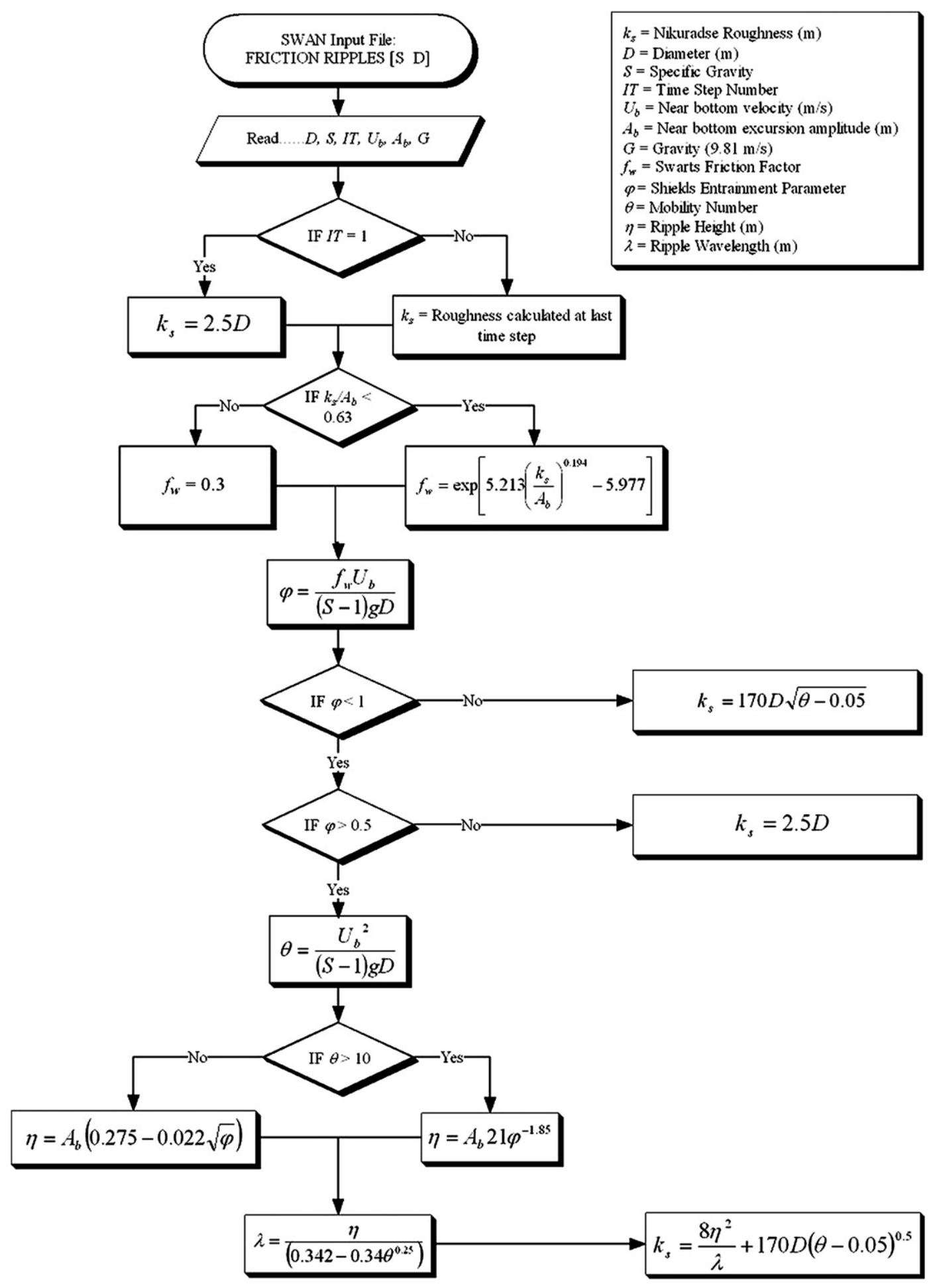

Fig. 2. Nielsen friction routine algorithm implemented into the SWAN model as a new subroutine.

model is based on the wave action balance equation (or energy balance in the absence of currents) with sources and sinks" (Booij et al., 2008):

$\frac{\partial N}{\partial t}+\frac{\partial}{\partial x}\left(c_{x} N\right)+\frac{\partial}{\partial y}\left(c_{y} N\right)+\frac{\partial c_{\sigma} N}{\partial \sigma}+\frac{\partial c_{\theta} N}{\partial \theta}=\frac{S_{t o t}}{\sigma}$.

The first term is the rate of change of action density with respect to time. The second and third term represent the propagation of the group velocity over a cartesian grid ( $c_{x}$ and $c_{y}$ are the group velocity in $x$ and $y$ space). The fourth term accounts for the shifting of the relative frequency of waves due to the effects of depth and currents. The fifth term is the refraction and propagation due to the depths and currents in the directional space ( $c_{\sigma}$ and $c_{\theta}$ are in spectral space). The right side of the equation incorporates $S_{\text {tot }}$, which is the total of all source and sink terms in the form of energy density, $E(\sigma, \theta)$.

The source and sink terms represent all "physical processes which generate, dissipate, and redistribute wave energy" (Booij et al., 2008). The terms in the source and sink Eq. (13) include the energy input from wind $\left(S_{i n}\right)$, energy redistribution due to non-linear interactions 


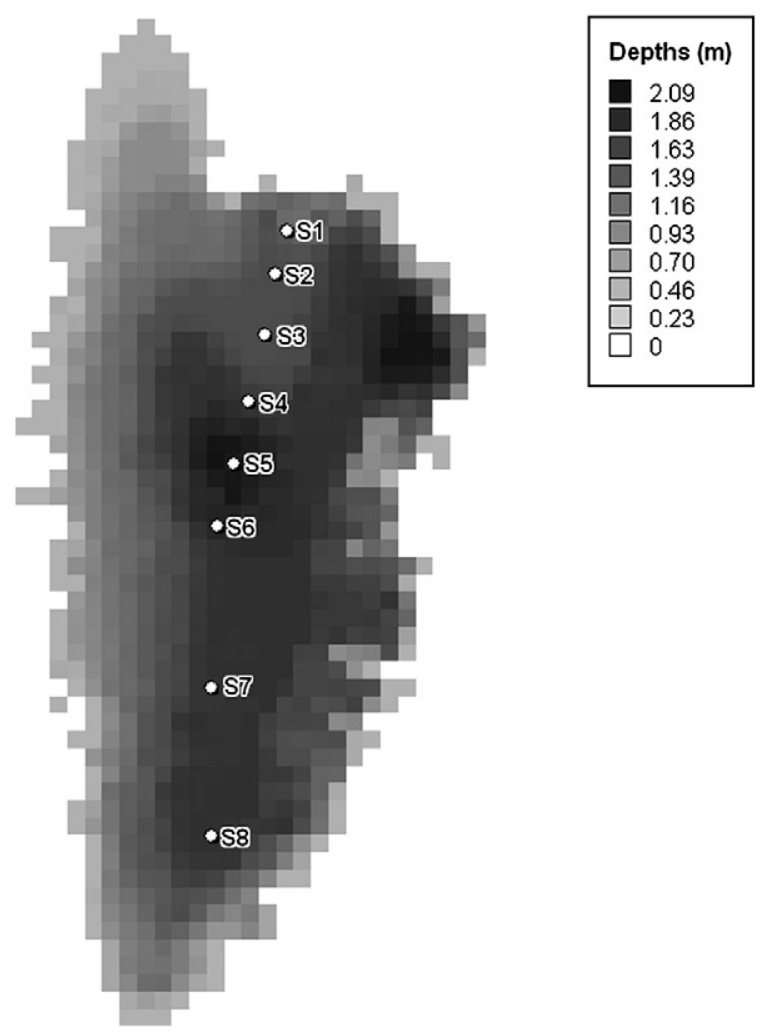

Fig. 3. Lake George bathymetry. The eight locations across the lake identify where observed data exists for validation.

$\left(S_{n l}\right)$, dissipation due to whitecapping $\left(S_{d s, w}\right)$, dissipation due to wave breaking $\left(S_{d s, b r}\right)$, and dissipation due to bottom friction $\left(S_{d s, b}\right)$.

$S_{t o t}=S_{i n}+S_{n l 3}+S_{n l 4}+S_{d s, w}+S_{d s, b r}+S_{d s, b}$.

\subsection{Bottom friction}

As a wave propagates into relatively shallow water, the orbital motion of the water particles extends down to the seabed. The interaction between the water particles and the seabed results in energy dissipation (e.g. Luo and Monbaliu, 1994) as described by Eq. (14)

$S_{d s, b}=-C_{b} \frac{\sigma^{2}}{g^{2} \sinh ^{2} k d} E(\sigma, \theta)$

where $k$ is the wave number. The SWAN model allows for three alternative methods for the determination of $C_{b}$, - (a) according to Collins (1972), (b) Hasselmann et al. (1973), and (c) Madsen et al. (1988).

Table 1

Observed data station details coordinates and depths. The coordinate units are in metres from an arbitrary reference.

\begin{tabular}{llll}
\hline Station ID & X coordinate & Y coordinate & Depth $(\mathrm{m})$ \\
\hline S1 & 6088 & 18710.132 & 1.3910 \\
S2 & 5849 & 17713.132 & 1.6873 \\
S3 & 5615 & 16311.132 & 1.6467 \\
S4 & 5276 & 14768.132 & 1.6359 \\
S5 & 4952 & 13351.132 & 1.7310 \\
S6 & 4608 & 11910.132 & 1.7537 \\
S7 & 4510 & 8201.132 & 1.8487 \\
S8 & 4486 & 4757.132 & 1.7630 \\
\hline
\end{tabular}

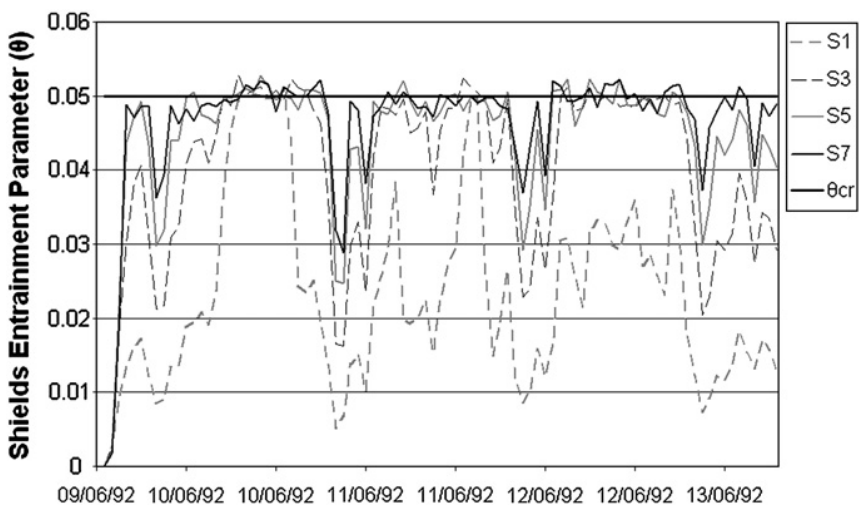

Fig. 4. Shields entrainment parameter for each of the eight output locations. The threshold value where ripples are expected to form is shown in bold at 0.05 . The time scale is from the 9th June to 13th June 1992.

The equation for determining $C_{b}$ as defined by Madsen incorporates a friction factor that was implicitly defined by Jonsson (1966) as:

$C_{b}=\frac{f_{w} g U_{b}}{2}$

The friction factor was also explicitly defined by Swart (1974) as defined in Section 1.1.

The present SWAN model assumes that the friction remains constant with respect to time. This formulation does not take into account the possibility of bedform transformations of a granular seabed whilst the seabed is subjected to wave action. A formulation that allowed for bedform variation would allow a friction coefficient that has a temporal variation.

\section{Case study: Lake George}

The first location for validation and testing of the new friction subroutine is Lake George, Australia. Lake George is located in the state of New South Wales. It covers an area of about $65 \mathrm{~km}^{2}$ and has a maximum depth of $2.1 \mathrm{~m}$. The advantage of using a shallow lake for investigating the impact of bedforms on wave evolution is that all developed waves will exhibit finite depth characteristics (Young and Babanin, 2006). Also, the Lake George bed is flat which makes the bottom topography simple and results will not be affected by a complex bathymetry. Sediment from this lake has been utilised in laboratory measurements to evaluate the interaction of the lake bed and waves (Babanin et al., 2005) (Fig. 3).

The observed data exists for eight stations located at various intervals spanning the length of the lake. The data timescale is an

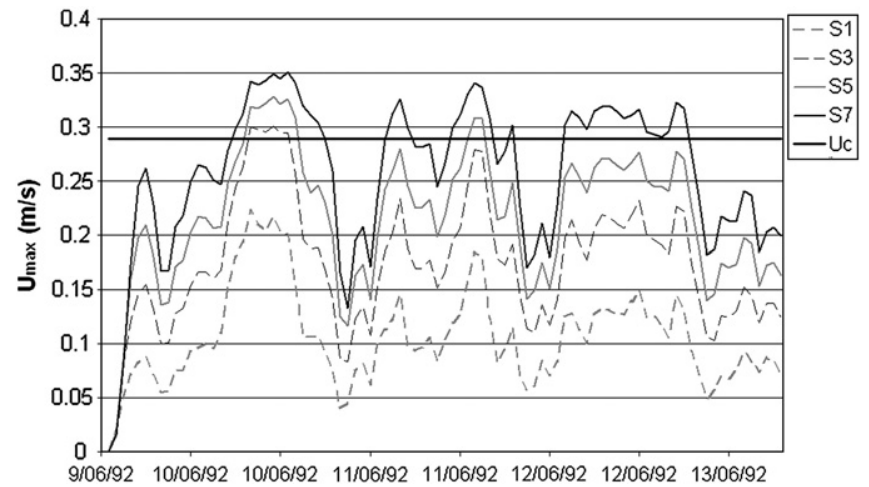

Fig. 5. The maximum orbital velocity for each of the eight locations. The threshold value where ripples are expected to form is shown in bold at $0.289 \mathrm{~m} / \mathrm{s}$. The time scale is from the 9th June to 13th June 1992. 

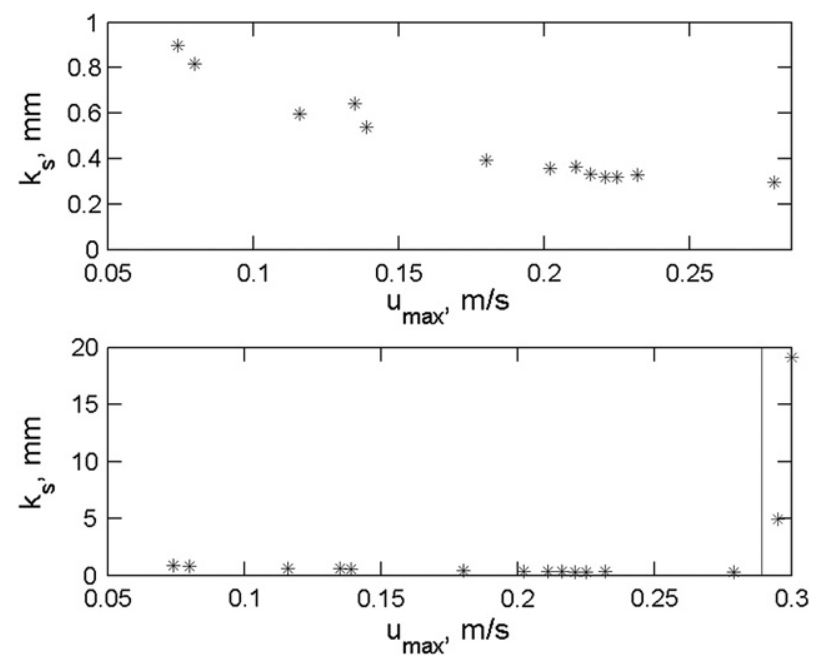

Fig. 6. Roughness coefficients obtained while the silt from Lake George was subjected to a range of unidirectional velocities.

18 month period from the 6th of March 1992 to 7th October 1993. This data was collected during an investigation on finite-depth spectral evolution as described in Young and Verhagen (1996) and Young et al. (1996) (Table 1).

The validation of the new friction subroutine involves three parts:

- Validating the algorithm, ensuring that ripples are found to occur at expected conditions.

- Analysis of the calculated roughness coefficient to ensure it's within the empirically known limits.

- Comparison of modelled wave parameters with the observed values.

\subsection{Algorithm validation}

Previous laboratory measurements using sediment from Lake George found that ripples begin to form when the free-flow velocity reaches $0.289 \mathrm{~m} / \mathrm{s}$ (Babanin et al., 2005). To validate the algorithm, the SWAN model was setup to output the maximum near bottom orbital velocity at each location to indicate when the threshold of $0.289 \mathrm{~m} / \mathrm{s}$ was reached, and output the Shields entrainment parameter, Eq. (4), at each location to indicate when the parameter is within the empirically defined ripple generation zone (between 0.05 and 1 ).
To validate the use of the Shields entrainment parameter, a period of strong winds was required to produce wave conditions high enough to result in ripple development. A period in June of 1992 was chosen as it encompassed the highest average wind speed for the 1992/1993 period averaging $10.4 \mathrm{~m} / \mathrm{s}$ and peaking at $17.71 \mathrm{~m} / \mathrm{s}$. This magnitude of the wind forcing was strong enough to initiate the sediment movement. Initial testing for another wind event that yielded an average of $5.2 \mathrm{~m} / \mathrm{s}$ (roughly the mean annual wind speed) was shown not to have sufficient energy to reach the Shields entrainment threshold of 0.5 , resulting in a lack of sediment movement.

Fig. 4 (in terms of the Shields criterion) and Fig. 5 (in terms of the velocity criterion) show identical periods of time where the threshold indicator for sediment mobility is exceeded. This indicates the existence of ripples according to Babanin et al. (2005), which supports and validates the theory of using Shields entrainment parameter as an indicator for ripple generation.

Fig. 4 also reveals a technical problem of implementing the rippleformation routine. It shows that once the threshold has been exceeded, the higher dissipation from the ripple bedform lowers the wave energy; hence on the subsequent time step the energy moves below the threshold value. This creates the zig-zag effect that can be observed along the threshold line. The issue was addressed by using the averaging technique that is discussed in Section 2.3 and smooths out the transitions between different bedform states.

\subsection{Grain size and roughness coefficient}

Laboratory experiments were conducted in Babanin et al. (2005) using silt from Lake George to determine a series of friction factors based on the Nikuradse roughness. Bed shear stress was measured using a shear plate which was subjected to unidirectional flow that ranged from $0.074 \mathrm{~m} / \mathrm{s}$ to $0.3 \mathrm{~m} / \mathrm{s}$. The roughness coefficient was found to grow rapidly by a factor of 60 when the velocity reached the threshold for which ripples are expected to form. This event can be observed in the last two data points of Fig. 6 after the vertical line that symbolizes the threshold for ripple formation.

Fig. 6 shows that the Nikuradse roughness coefficients determined in Babanin et al. (2005) were found to range from a minimum of $0.00032 \mathrm{~m}$ in the absence of ripples, up to a maximum of $0.0191 \mathrm{~m}$ when ripples form. Without explicit grain size diameter measurements, the minimum roughness value can be used in conjunction with Eq. (2) to provide an estimation of the grain diameter. The specific gravity can be assumed to be 2.65 for sediment comprised mostly of quartz.

To validate the Nikuradse roughness coefficient that is generated at each time step, the model was setup to output the roughness value for
Location 3 - Default Friction Subroutine

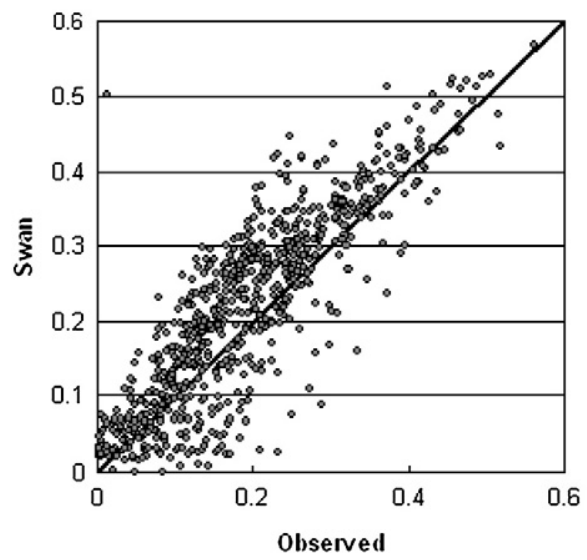

Location 3 - Ripple Friction Subroutine

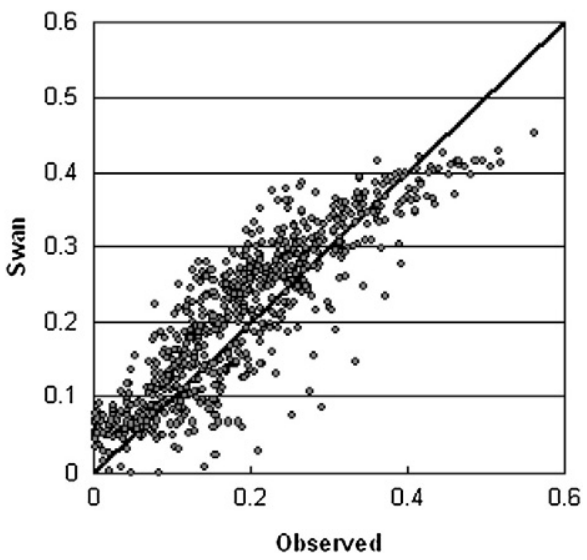

Fig. 7. Scatter plot of significant wave height $(\mathrm{m})$ for Location 3 showing under-prediction for higher wave conditions. 
Table 2

Pseudocode algorithm for the averaging technique discussed in Section 2.3.

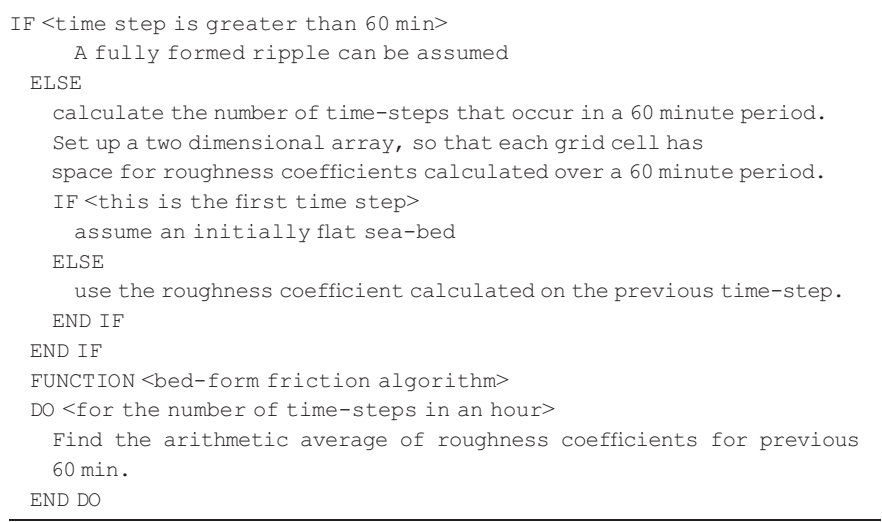

every location and time step for the same period of time in June of 1992 that encompassed the strong wind event. The analysis of the coefficient time series showed that the roughness varied from $0.00032 \mathrm{~m}$ to $0.02 \mathrm{~m}$. This range corresponds extremely well with the minimum and maximum ranges of $0.00032 \mathrm{~m}$ and $0.0191 \mathrm{~m}$ found experimentally for Lake George silt (Babanin et al., 2005). This provides an indirect quantitative verification of the new routine incorporated in the SWAN model.

\subsection{Model accuracy}

To evaluate the effects of the additional friction algorithm into the SWAN model, and the associated accuracy achieved when compared to default friction routines, a model was setup for Lake George to run for the full duration of October 1992. The month of October was selected because the observed data shows that October has the highest average of wind speeds of any month. Results from the algorithm validation (Section 2.1) indicated that for Lake George, sediment movement was only expected in high energetic conditions.

SWAN was setup as a two-dimensional non-stationary model over a Cartesian grid with the nautical convention for wind/wave direction. Wind input was assumed spatially constant over the lake with a 60-minute time step. The computational time step was set to $7.5 \mathrm{~min}$. Functions and parameters absent from the aforementioned description are set to default options built into the SWAN model.

Initial testing shows an improved correlation between the new friction subroutine and the observed data for significant wave height. However an erroneous under prediction became prevalent for waves with a significant wave height greater than $0.4 \mathrm{~m}$ (Fig. 7). The cause of this attenuation is an over-estimation of the roughness coefficient at high-sea conditions, which became apparent during the algorithm validation as in Section 2.1. The calculated ripples that are formed during the high energy events assume a fully developed ripple for that energy/ wave condition. In reality, a ripple takes roughly $60 \mathrm{~min}$ to form, and 100 min to become fully developed based on studies by Davis (2005) where a fine sediment with a $D_{50}$ ranging from $0.2 \mathrm{~mm}$ to $0.6 \mathrm{~mm}$ were subjected to an irregular wave field. The degradation of a ripple is due to microbiological activity, and it has been shown to take 4-6 h to degrade back to flat bed conditions (Amos et al., 1988).

To combat this issue, the model was altered to "remember" the previous hours worth of friction coefficients, as the ripple dimensions are dependent on both the existing and previous hydrodynamic conditions (Monbaliu et al., 2007). This history was used to provide an arithmetic average of the previous friction coefficients for the current time-step (Table 2). The effect of this alternative methodology showed an improvement in prediction for capturing the few peak waves, which can be observed in the scatter plots but does not alter the data significantly to impact the RMS error.

Another aspect that can be observed in the output data from the model is related to wind direction. The hypothesis of this paper (that a model using a friction coefficient that does not incorporate the roughness growth due to ripples results in the bottom friction dissipation term being the dominate contributor to the modelling error in finite depth applications) should manifest itself in the model output as an inaccuracy that increases as a function of fetch for directional homogenous wind events that sustain over a period of time. The station location with the greater fetch for the particular wind direction is proportional to the magnitude of the model inaccuracy. The wave-height overestimation that increases with fetch is an example of the impact of consistently underestimating the friction factor in the model. Wave energy propagates across the lake accumulating a marginal error at each grid cell that eventually combines into a significant error. It should be noted that this phenomenon is a separate issue to a similar analysis discussed in Breugem and Holthuijsen (2005), which details how northerly and southerly winds at Lake George deviate from an idealised situation of an infinitely wide fetch when the dominating wind direction is from the south (due to the unique triangular shape of the lake).

Fig. 8 depicts the results at station 6 , which was chosen for its central location at Lake George. The model improvement resulting from the new friction subroutine is reflected in the least squares error defined on Fig. 8 for the correlation between the default friction subroutine and the observed data, and the new additional subroutine and the observed data. The correlation coefficient is also shown, but
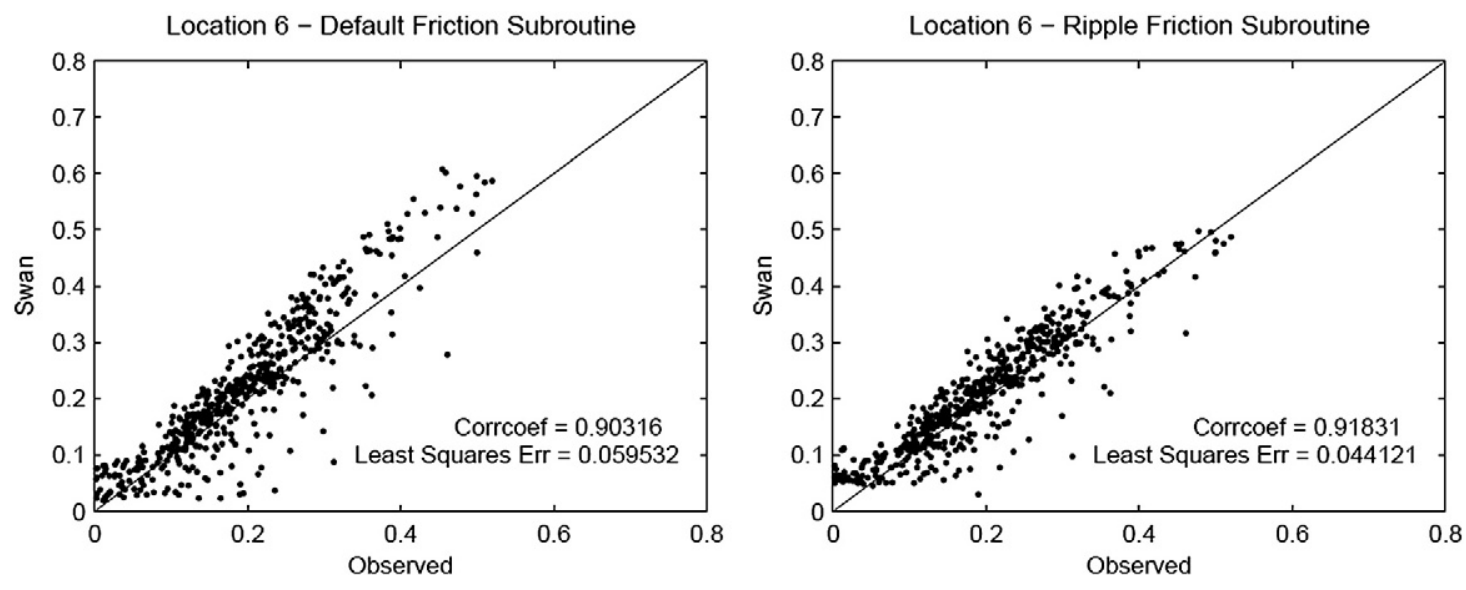

Fig. 8. Scatter plot of significant wave height (m) for Location 6. 
Table 3

RMS error for significant wave height and peak period for all stations at Lake George.

\begin{tabular}{|c|c|c|c|c|c|c|}
\hline Station & $H_{s}$ default & $H_{s}$ ripples & Percentage improvement (\%) & $T_{p}$ default & $T_{p}$ ripples & Percentage improvement (\%) \\
\hline 1 & 0.100 & 0.090 & 10.56 & 1.219 & 1.219 & -0.02 \\
\hline 2 & 0.076 & 0.068 & 10.79 & 0.332 & 0.324 & 2.50 \\
\hline 3 & 0.058 & 0.051 & 11.22 & 0.741 & 0.743 & -0.28 \\
\hline 4 & 0.059 & 0.047 & 20.80 & 0.263 & 0.269 & -2.28 \\
\hline 5 & 0.059 & 0.043 & 26.25 & 0.258 & 0.267 & -3.68 \\
\hline 6 & 0.060 & 0.044 & 25.89 & 0.259 & 0.251 & 3.28 \\
\hline 7 & 0.094 & 0.072 & 23.14 & 0.306 & 0.283 & 7.51 \\
\hline \multirow[t]{2}{*}{8} & 0.114 & 0.091 & 20.12 & 0.758 & 0.727 & 4.04 \\
\hline & & Average Improvement (\%) & 18.60 & & Average Improvement (\%) & 1.38 \\
\hline
\end{tabular}

does not give an accurate representation of the model accuracy as it illustrates the correlation of the linearity of the prediction only. The least squares error, Eq. (16) is a representation of the accuracy of a 1:1 linear relationship between the model data and the observed data:

$R=\sqrt{\frac{\sum_{i=1}^{n}\left(y_{i}-f(x, \beta)\right)^{2}}{n}}$

where $y_{i}$ represents the observed data and $f(x, \beta)$ is the model output.

The full details for the least squares error at all station locations are shown in Table 3. There was an overall improvement for the prediction of a significant wave height at all eight locations at Lake George, with an average percentage improvement of \%18.6, and a maximum improvement of \%26.26. The model improvement for the peak period hindcasting was minor, with most stations exhibiting a percentage improvement of $\pm \% 4$ or less.

Some amount of uncertainty exists for the modelling results at Location 7 (which can be observed in the additional model overestimation in Fig. 9), although the results do exhibit an improved correlation between the model results based on the ripple friction subroutine and the observed data. The correlation for the peak period at Location 7 also shows an improvement in the scatter plot, shown in Fig. 10.

\section{Case study: Lakes Entrance}

The previous section validated the ripple friction algorithm based on laboratory testing of sediment samples from Lake George and field measurement in Lake George. It should be stressed that the newly implemented bottom-friction routine described in Section 1.1 and Fig. 2 makes this friction dependent not only on the presence/absence of ripples, but also on the grain size of the bottom sediment. The laboratory measurements of Babanin et al. (2005) provided the Nikuradse roughness for the Lake George sediment, and the routine worked well

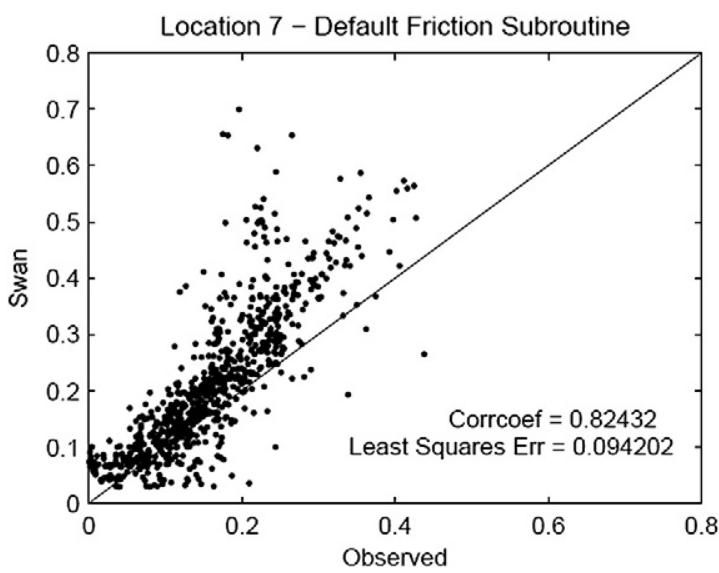

for this given sediment. Further testing of the routine is best done by applying the routine to a site with a different grain size (Table 4).

The ripple friction subroutine was subsequently field tested using the observed data from a different site - offshore from Lakes Entrance, Victoria. Not only are the wave conditions different at Lakes Entrance, so is the bottom sediment. Sediment characteristics are explicitly known at this location and the wave activity incorporates longer period waves from the open ocean.

Lakes Entrance is a coastal town located along the south east coast of Victoria, Australia, between Bairnsdale and Orbost. A man made channel was completed in 1889 to connect Bass Strait to the Gippsland lakes (Kowarsky, 2007).

The Victorian coast is subjected to long period waves that are generated from the Southern Ocean, Pacific Ocean, and the Tasman Sea. A proportion of these waves will refract around Tasmania and travel across Bass Strait until they meet the Victorian coastline. To capture all possible wave generation that can affect the coast of Lakes Entrance, the coarse grid used for the first level of modelling incorporated a large area surrounding Australia. Two further grid nesting's were employed to focus the model on the coastal region surrounding Lakes Entrance as shown in Fig. 11.

A wave rider buoy located $2.3 \mathrm{~km}$ off the coast of Lakes Entrance provides observational wave data for height, direction and period at 20 min intervals for the year 2008 (Oldfield, 2007). The approximate depth at the wave rider buoy is $16.3 \mathrm{~m}$.

The SWAN model was driven with winds obtained from the Australian Bureau of Meteorology numerical weather prediction models. The outer coarse grid employed winds from the Global Analysis and Prediction (GASP) model that has a grid resolution of $1^{\circ}$ at 3-hour intervals. The inner finer resolution grids utilised winds from Mesoscale Limited Area Prediction System (MesoLAPS) model with the grid resolution of $0.125^{\circ}$ at 1 -hour intervals.

The coarse grid was run for the year of 2008 with output locations near the east and west entrances to Bass Strait. Note that only one set of sediment parameters are accepted in the SWAN setup file. The ripple

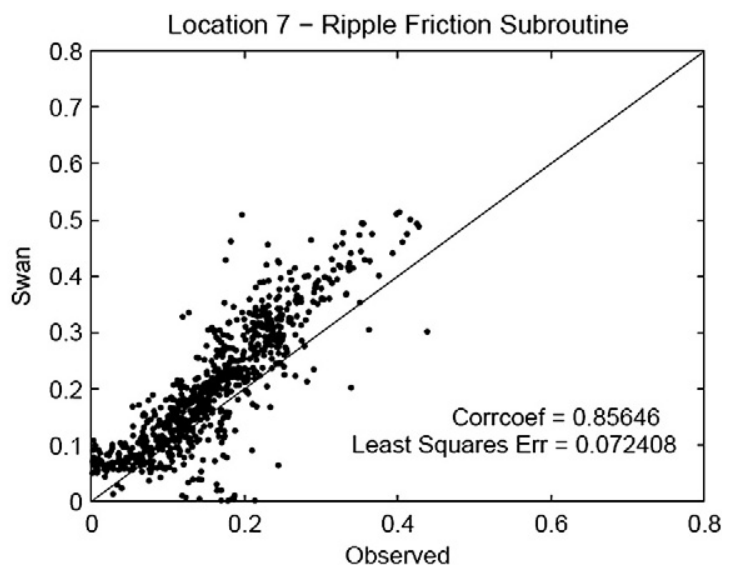

Fig. 9. Scatter plot for significant wave height (m) at Location 7. 

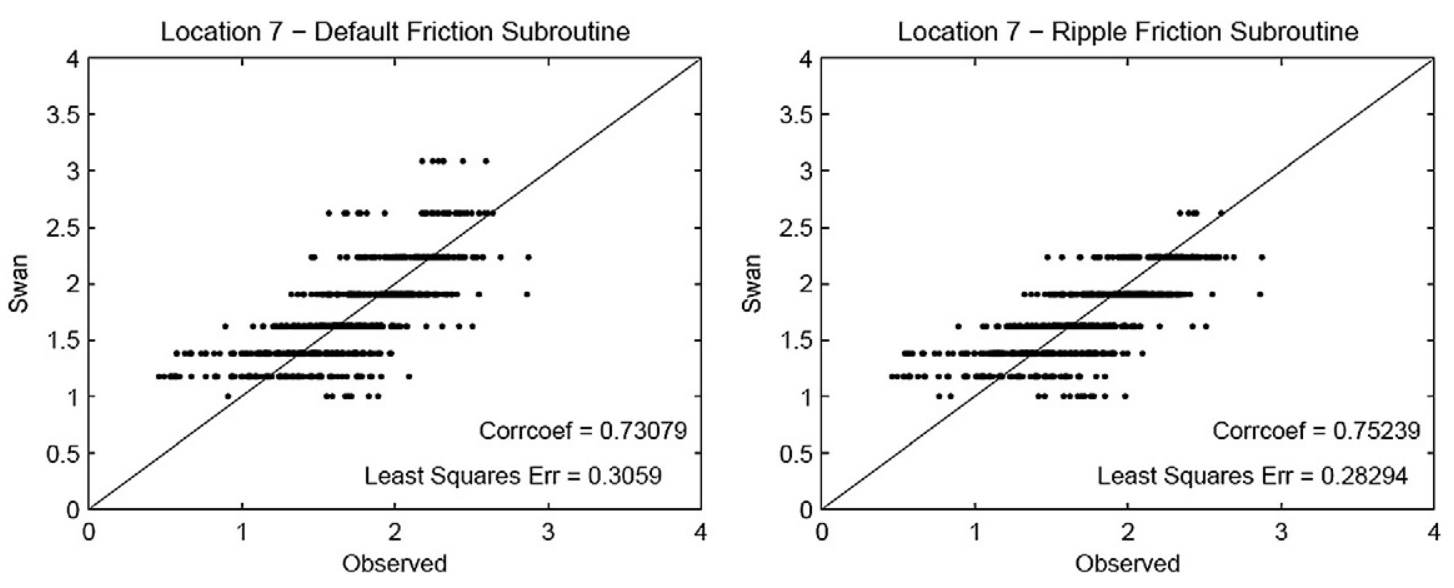

Fig. 10. Scatter plot for peak period (s) at Location 7.

algorithm should only be switched on for localised grid runs where ripple formation may occur, as the sediment parameters cannot be assumed universal over large regions. The occurrence of ripples is only relevant for the level three grid, approaching Lakes Entrance.

The fine resolution of the nested grids required a smaller time step which is computationally expensive and time consuming. Therefore, rather than simulating the full 2008 year a smaller snapshot of time was selected. The results from the output locations derived from the level one model run showed high energetic conditions occurring during the month of October. The results of the corresponding level three model run are shown in Fig. 12.

The time series shows that the model results for the default friction in the SWAN performed well, and the results from the ripple friction routine provided very similar results. The two versions of the model can be observed to deviate from one another at certain periods, although the least squares error was identical for both (0.26). The error for both models is small and is in the range of the typical error magnitude that is acceptable within the limitation models of this nature as well the as errors associated with measuring metocean data.

It should be noted that the peak that occurs around the 7th of October is much larger than the observed data due to inaccurate wind model data during this period of time. The inaccuracy is observed when comparing the mesoLAPS model winds to the anemometer winds recorded at Lakes Entrance for this time period. For the month of October 2008, this is the only obvious discrepancy that is apparent in the wind data.

Further analysis of the calculated roughness coefficients in the friction ripple routine model run shows that, at this particular location at Lakes Entrance, the algorithm determined that ripple evolution only occurred at a limited number of time steps. These ripples occurred during the two larger events around the 9th and 23rd of October. Therefore during most roughness coefficient calculations during October 1992, the roughness was based on sediment grain size only, as per Eq. (2).

This validates the implementation of the friction routine in locations where ripples are not present, and the Nikuradse roughness is based on the sediment diameter alone. This is important for applications where the presence of ripple bed-forms are unknown, thus requiring an

Table 4

Sediment properties for Lakes Entrance (Kowarsky, 2007).

\begin{tabular}{llll}
\hline Lakes Entrance dredging zones & D50 $(\mathrm{mm})$ & Mean $(\mathrm{mm})$ & Specific gravity \\
\hline The Bar & 0.41 & 0.40 & 2.6 \\
The Entrance & 0.43 & 0.41 & 2.6 \\
\hline
\end{tabular}

algorithm that represents flat sea beds with an appropriate friction factor.

The likelihood of ripples in a coastal application is spatially dependent, and the accuracy of the model is influenced by the bottom friction dissipation experienced by the shoaling waves as they propagate towards a model output location. For the Lakes Entrance case study, it is unlikely that ripples have had much of an opportunity to impact on the waves moving towards the particular output location through the deeper ocean. There would possibly be more influence as the waves enter shallower regions, but without observed data it is impossible to ascertain the model performance elsewhere.

\section{Conclusions}

A bottom-friction routine, based on the Nielsen algorithm, was introduced into the SWAN model which makes the bottom friction for waves dependent on the presence/absence of ripples if the sea bed is mobile, and on the grain size of the sediment. The routine is suitable for the spectral models of the wave evolution, and in the present study it was tested by means of the SWAN model by hindcasting waves at two finite-depth field sites.

Initial modelling of Lake George using the default friction configuration in the SWAN model showed that the significant wave height was overestimated at most time steps (in some locations the predicted wave height was more than double the observed data). This discrepancy supports the hypothesis that in the shallow depths when friction dissipation is the dominating dissipation term, an overestimation of wave energy will occur when conditions and sediment characteristics are likely to produce sand ripples.

The results from the Lake George SWAN model using the ripple friction algorithm case are very promising (the water depth is approximately $2 \mathrm{~m}$ across the lake). In some locations a model agreement with the observed data was excellent. There was an overall improvement in model prediction for significant wave height, and a small yet identifiable improvement on the peak period. Limitations in verification exist due to the shallow nature of the lake, the presence of only relatively small waves, and corresponding short wave periods.

The algorithm that determines the occurrence of sediment mobility and the evolution of bed-forms was also validated to emulate the expected behaviour found in laboratory experiments using a sediment sample from Lake George (Babanin et al., 2005). The near bed orbital velocity exceeded the uni-directional velocity found in laboratory experiments (that was deemed to be the threshold to initiate sediment mobility) at similar time steps as the Shields parameter threshold predicted. The maximum roughness coefficient calculated by the algorithm for the month of October 1992 was 


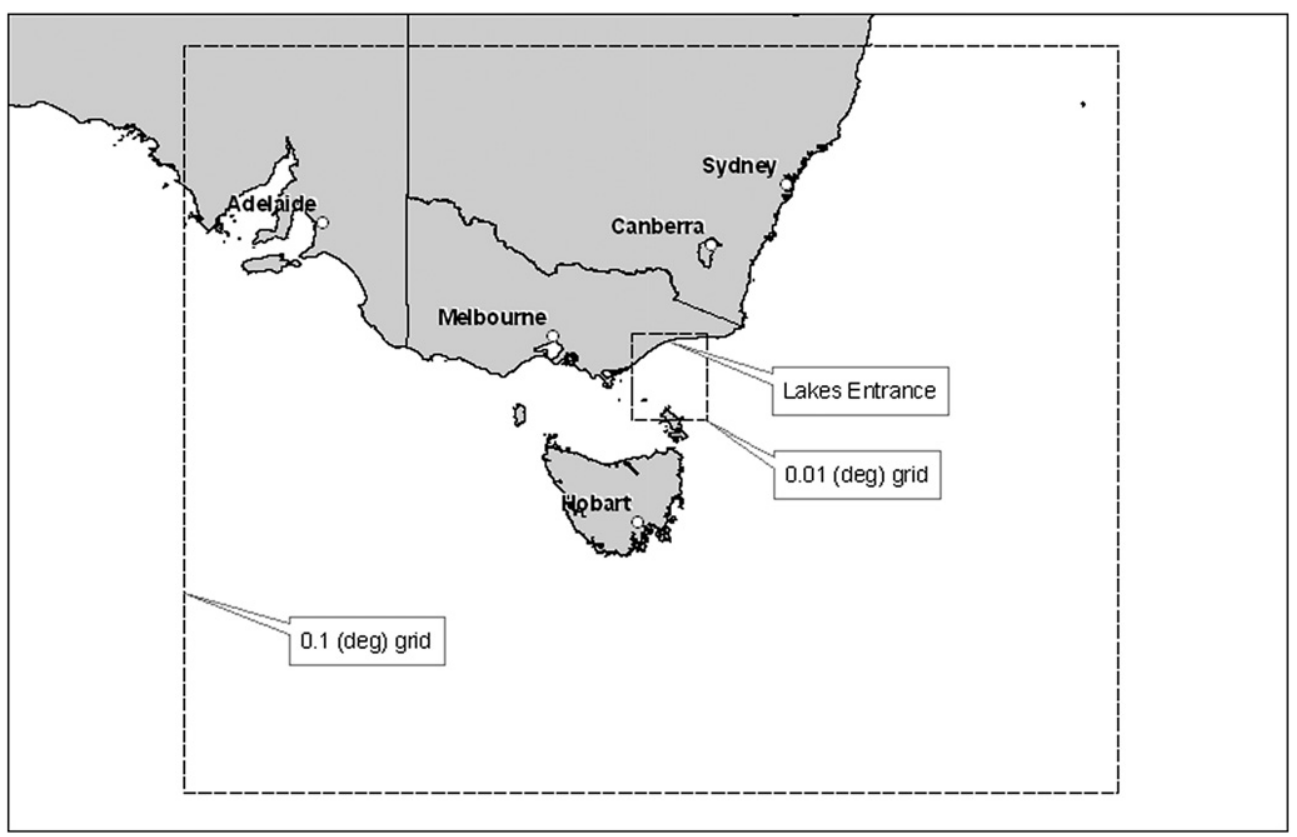

Fig. 11. Bathymetry inner nesting grid extents for Lakes Entrance model.

$19.1 \mathrm{~mm}$, which is similar to the maximum value of $20 \mathrm{~mm}$ expected to occur for sediment at Lake George (Babanin et al., 2005).

An overestimation of the presence of fully developed ripples was amended by implementing an averaging process that remembers the history of the roughness at each grid cell. The final roughness factor at any given time step and location was derived from this array.

The conditions experienced at Lake George were too low to validate the event of extremely high energy that would cause any bed-forms to be "washed out" or flattened. The Shields entrainment parameter validation in Section 2.1 could be assumed to provide a similar result for extremely high energetic conditions based on the similar shape of the entrainment parameter and orbital velocity time series graphs shown in Figs. 4 and 5 respectively.

Further testing was done for an offshore coastal location at Lakes Entrance at a depth of $16.3 \mathrm{~m}$. The original SWAN Model showed quite acceptable results. At the particular output point in question, there were no ripples found to exist based on the bed-form algorithm. The roughness coefficient was therefore solely based on the grain size diameter. The results from both models are comparable as they both utilised a constant value for roughness over the model run due to the absence of bed-forms. Since the Lakes Entrance sand size $(0.41 \mathrm{~mm})$ is very different from the Lakes George silt size $(0.13 \mathrm{~mm})$ this outcome provides a support to the grain-size dependence of the new bottom-friction routine (Babanin et al., 2001).

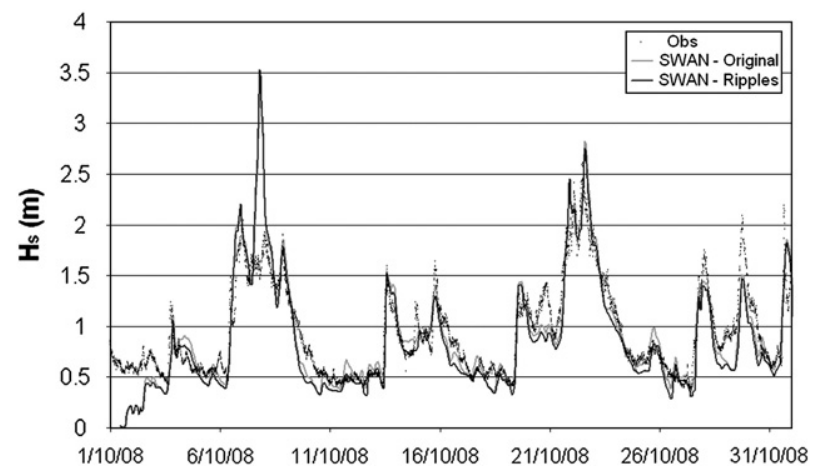

Fig. 12. Time series for Lakes Entrance showing observed data and model results for October 2008.

\section{References}

Amos, C., Bowen, A. Huntley, D., Lewis, C., 1988. Ripple generation under the combined influences of waves and currents on the Canadian continental shelf. Cont. Shelf Res. 8 (10), 1129-1153.

Babanin, A.V., Young, I.R., Banner, M.L., 2001. Breaking probabilities for dominant surface waves on water of constant depth. J. Geophys. Res. 106 (C6), 11659-11676.

Babanin, A.V., Young, I.R., Mirfenderesk, H., 2005. Field and laboratory measurements of wave-bottom interaction. Coasts and Ports: Coastal Living - Living Coast. Institution of Engineers, Australia Adelaide, Australia.

Booij, N., et al., 2008. Swan technical documentation: Swan cycle III version 40.72. Delft University of Technology.

Booij, N., Ris, R.C., Holthuijsen, L.H., 1999. A third-generation wave model for coastal regions, 1, model description and validation. J. Geophys. Res. 104 (C4), 7649-7666.

Breugem, W.A., Holthuijsen, L.H., 2005. Generalised shallow water wave growth from Lake George without the effect of fetch width. J. Waterway, Port, Coastal, and Ocean Engineering ASCE.

Collins, J.I., 1972. Prediction of shallow water spectra. J. Geophys. Res. 77 (15), 2693-2707.

Davis, J.P., 2005. A Spectral Approach to the Transient Analysis of Wave-Formed Sediment Ripples. The University of Adelaide, Adelaide.

Grant, W.D., Madsen, S.O., 1982. Movable bed roughness in unsteady oscillatroy flow. J. Geophys. Res. 87(No. C1), 469-481.

Hasselmann, K., et al., 1973. Measurements of wind-wave growth and swell decay during the Joint North Sea Wave Project (JONSWAP). Dtsch. Hydrogr. Z. Suppl. 12 (A8).

Hsiao, S.V., Shemdin, O.H., 1978. Bottom dissipation in finite-depth water waves. 16th Int. Conf. Coastal Eng. ASCE, Hamburg, pp. 434-448.

Jonsson, I.G., 1966. Wave boundary layers and friction factors. Proc. 10th Int. Conf Coastal Engineering ASCE, pp. 127-148.

Komen, G.J., et al., 1994. Dynamics and Modelling of Ocean Waves. Cambridge University Press, Cambridge.

Kowarsky, J., 2007. Sediment Sampling and Analysis Plan: Lakes Entrance Sand Management Program, Gippsland Ports, Lakes Entrance.

Lettau, H., 1969. Note on aerodynamic roughness parameter estimation on the basis of roughness element description. J. Appl. Meteorol. 8, 828-832.

Luo, W., Monbaliu, J., 1994. Effects of the bottom friction formulation on the energy-balance for gravity-waves in shallow-water. J. Geophys. Res. Oceans 99 (C9), 18501-18511.

Madsen, O.S., Poon, Y.K., Graber, H.C., 1988. Spectral wave attenuation by bottom friction: theory. Proc. 21st Int. Conf. Coastal Engineering. ASCE, pp. 492-504.

Melnikhova, O.N., Volkov, P.Y., 2000. Sediment transport by eddies formed in the boundary layer of open flows. New Trends in Water and Environmental Engineering for Safety and Life. .

Miller, M.C., Komar, P.D., 1980. A field investigation of the relationship between oscillation ripple spacing and the near bottom water orbital motions. J. Sediment. Petrol. 50 (No. 1), 183-191.

Mirfenderesk, H., 1999. The Dissipation of Ocean Wave Spectra due to Bottom Friction. University of New South Wales.

Monbaliu, J., Ardhuin, F., Wolf, J., 2007. Bottom dissipation. Prog. Oceanogr. 75 (4), 636-640.

Nielsen, P., 1981. Dynamics and geometry of wave-generated ripples. J. Geophys. Res. 86 (C7), 6467-6472.

Nielsen, P., 1992. Coastal Bottom Boundary Layers and Sediment Transport. Advance Series on Ocean Engineering. World Scientific. 
Oldfield, D., 2007. Monitoring of Trials, Gippsland Ports.

Raudkivi, A.J., 1988. The roughness height under waves. J. Hydraul. Res. 26, 259-584.

Riedel, P., O'Brien, P., Townsend, M., 2005. The effect of ocean swell on the stability of Adelaide's metropolitan beaches. Coastal Living - Living Coast. Institution of Engineers, Australia Adelaide, Australia, Coasts and Ports.

Sato, S., Mitani, D., 1988. Geometry of sand ripples and net sand transport rate due to regular and irregular oscillatory flows. Coast. Eng. Jpn 30 (No. 2), 89-98.

Swart, D.H., 1974. Offshore Sediment Transport and Equilibrium Beach Profiles.

Swart, D.H., 1976. Predictive equations regarding long shore transport. 15th Conference on Coastal Engineering, 2, pp. 1113-1132.
Young, I.R., Babanin, A.V., 2006. The form of the asymptotic depth-limited wind wave frequency spectrum. J. Geophys. Res. 111 (C06031). doi:10.1029/2005JC003398, 15p. Young, I.R., Gorman, R.M., 1995. Measurements of the evolution of ocean wave spectra due to bottom friction. J. Geophys. Res. 100 (C6), 10987-11004.

Young, I.R., Verhagen, L.A., 1996. The growth of fetch limited waves in water of finite depth. Part 1. Total energy and peak frequency. Coastal Eng. V(29), 101-121.

Young, I.R., Verhagen, L.A., Khatri, S.K., 1996. The growth of fetch limited waves in water of finite depth. Part III: directional spectra. Coastal Eng. 29, 101-121. 